\title{
Expression of CD24, P-cadherin and S100A4 in tumors of the ampulla of Vater
}

\author{
Daniel Baumhoer ${ }^{1,7}$, Marc-Oliver Riener ${ }^{2,7}$, Inti Zlobec ${ }^{1}$, Luigi Tornillo ${ }^{1}$, \\ Alexander Vogetseder ${ }^{2}$, Glen Kristiansen ${ }^{2}$, Wolfgang Dietmaier ${ }^{3}$, Arndt Hartmann ${ }^{4}$, \\ Peter H Wuensch ${ }^{5}$, Fausto Sessa ${ }^{6}$, Petra Ruemmele ${ }^{3}$ and Luigi M Terracciano ${ }^{1}$
}

\begin{abstract}
${ }^{1}$ Institute of Pathology, University of Basel, Basel, Switzerland; ${ }^{2}$ Institute of Surgical Pathology, University of Zürich, Zürich, Switzerland; ${ }^{3}$ Institute of Pathology, University of Regensburg, Regensburg, Germany; ${ }^{4}$ Institute of Pathology, University Erlangen, Erlangen, Germany; ${ }^{5}$ Institute of Pathology, Municipal Hospitals of Nuremberg, Nuremberg, Germany and ${ }^{6}$ Anatomic Pathology Unit, Department of Human Morphology, University of Insubria, Varese, Italy
\end{abstract}

\begin{abstract}
Carcinomas of the Vaterian system are rare and presumably arise from preexisting adenomas (adenomacarcinoma-sequence). Usually, biopsies are obtained to confirm and specify endoscopic findings, but differentiating reactive atypia from dysplasia or dysplasia from invasive carcinoma can sometimes be difficult or even impossible on morphological criteria alone. In case of invasive carcinoma, furthermore, the precise classification of carcinoma subtypes needs to be established since the distinct subtypes differ significantly in terms of clinical outcome. The cell adhesion proteins CD24, P-cadherin and S100A4 were shown to be expressed in several carcinomas and in dysplastic epithelium but only rarely in normal mucosa. We therefore investigated their expression in 177 carcinoma, 114 adenoma and 152 normal mucosa specimens of the ampulla of Vater. Although the expression of the cell adhesion proteins did not differ between the carcinoma subtypes, marked differences between normal mucosa, adenoma and carcinoma samples were observed. All marker proteins were expressed in less than 7\% of normal mucosa samples (S100A4 in only $1 \%$ of cases) and showed an increasing expression from adenoma to invasive carcinoma. Our findings suggest that P-cadherin and S100A4 are helpful in discriminating normal mucosa or reactive atypia from neoplastic lesions. CD24 and S100A4, furthermore, can assist in the differential diagnosis of dysplasia vs invasive carcinoma.

Modern Pathology (2009) 22, 306-313; doi:10.1038/modpathol.2008.192; published online 28 November 2008
\end{abstract}

Keywords: ampullary carcinoma; ampulla of Vater; tissue microarray; CD24; P-cadherin; S100A4

Ampullary carcinomas are generally rare with an incidence of approximately 5.7 cases per million population per year, but are still more common than any other tumor of the small intestine. ${ }^{1-3}$ In up to $91 \%$ of cases, residual adenomas can be detected in the tumor periphery, indicating an adenoma-carcinoma sequence similar to that observed in colorectal cancer. $^{4,5}$ Patients commonly present early with obstructive jaundice due to neoplastic occlusion of the ampullary orifice. ${ }^{6}$ Early detection but also biologic differences are held responsible for the better prognosis of ampullary tumors following pancreaticoduodenectomy compared with other periampullary tumors (median survival 30-50

Correspondence: Dr D Baumhoer, MD, Institute of Pathology, University of Basel, Schönbeinstrasse 40, Basel 4003, Switzerland. E-mail: dbaumhoer@mac.com

${ }^{7}$ These authors contributed equally to this work.

Received 18 August 2008; revised 13 October 2008; accepted 19 October 2008; published online 28 November 2008 months, 5-year survival rate $21-64 \%) .{ }^{7-13}$ Histologically, several subtypes of ampullary carcinomas can be distinguished, obviously deriving from the different types of mucosa adjoining the Vaterian system. ${ }^{1,14}$ According to the classification by the Armed Forces Institute of Pathology (AFIP) an intestinal type, resembling carcinomas of intestinal origin and a pancreatico-biliary type, resembling carcinomas of the extrahepatic bile ducts or the pancreas are the most common subtypes and account for $70-95 \%$ of cases. ${ }^{14-18}$ Other subtypes include an intestinal-mucinous type, an invasive papillary type, a poorly differentiated adenocarcinoma and several rather uncommon entities. ${ }^{15}$ Importantly, the various subtypes have been shown to differ significantly in terms of prognosis, with a mean survival of 76 months for intestinal type compared to 41 months in pancreatico-biliary type carcinomas (Ruemmele et al, submitted).

CD24 and P-cadherin (cadherin-3) are cell adhesion proteins which have both been shown to be 
upregulated in several carcinomas and to be associated with a poor clinical outcome. ${ }^{19-24}$ Also intraand extrahepatic cholangiocarcinomas have been reported to express CD24. ${ }^{25-27} \mathrm{P}$-cadherin was found to be constantly upregulated in intrahepatic cholangiocarcinomas using cDNA microarray technique that was confirmed using immunohistochemistry. ${ }^{26}$ Interestingly, the expression of P-cadherin was only detected in carcinoma but not in normal mucosa samples. S100A4 is a calcium binding protein belonging to the S100 family and was proposed to be a promising new marker protein for dysplasias and carcinomas of the biliary tract. A recent study by Zhao and colleagues showed S100A4 expression in more than $80 \%$ of extrahepatic bile duct carcinomas and high-grade dysplasias and constant negativity in normal mucosa. ${ }^{28}$

Since pathologists generally receive bioptic material from ampullary tumors obtained during endoscopy, the differential diagnosis of reactive glandular atypia vs dysplasia and adenoma vs carcinoma can often be difficult or even impossible using conventional light microscopy only. Furthermore, accurate classification of carcinoma subtypes is of crucial importance due to significant differences in clinical outcome. We investigated a large cohort of ampullary tumors, including 177 carcinoma, 114 adenoma and 152 normal mucosa specimens for their expression of CD24, P-cadherin and S100A4 to determine potentially distinctive immunoprofiles for normal, dysplastic and malignant lesions and for carcinoma subtypes of the ampulla of Vater.

\section{Materials and methods}

\section{Tissue Samples and Patient Characteristics}

The files of the Institute of Pathology, University Hospital Basel (Switzerland), the Institute of Pathology, University of Regensburg (Germany), the Institute of Pathology, Municipal Hospitals of Nuremberg, and the Anatomic Pathology Unit, Department of Human Morphology, University of Insubria, Varese (Italy) were searched for adenomas or carcinomas of the ampulla of Vater over the period from 1985-2005. Only tumors derived from the periampullary duodenal mucosa, the common channel and the ampullary pancreatic and common bile duct were included in this study. According to the recommendations of the Armed Forces Institute of Pathology (AFIP) these tumors were considered collectively as 'ampullary' tumors. ${ }^{15}$ In total, 177 carcinoma, 114 adenoma and 152 normal mucosa samples from surgically resected cases were retrieved. Sufficient paraffin-embedded tissue for Tissue Microarray construction was available in all cases. The male to female ratio was $3: 2$, mean age at diagnosis was 63 years (range 15-81 years). Carcinoma samples included 19 pT1 (11\%), 59 pT2 (33\%), 63 pT3 (36\%), and 13 pT4 (7\%) tumors and
$17 \mathrm{G} 1$ (10\%), $82 \mathrm{G} 2(46 \%)$ and $55 \mathrm{G} 3$ (31\%) cases (no data concerning T-stage and grading in 23 samples). $73(41 \%)$ carcinoma patients were node-positive (Nstage) and 2 (1\%) had hematogenous metastases (Mstage) at initial diagnosis.

\section{Tissue Microarray Construction}

Tissue samples were fixed in buffered $4 \%$ formalin, embedded in paraffin, and used to construct a Tissue Microarray. Briefly, hematoxylin-eosin (H\&E) stained sections were made from each selected primary block (donor blocks) to define representative tissue regions. Tissue cylinders $(0.6 \mathrm{~mm}$ in diameter) were then punched from the region of the donor block with the use of a custommade precision instrument (Beecher Instruments, Silver Spring, USA). Tissue cylinders were transferred to a $25 \times 35 \mathrm{~mm}$ paraffin block to produce the Tissue Microarray block used for the study. The resulting block was cut into $3 \mu \mathrm{m}$ sections that were transferred to glass slides by use of the Paraffin Sectioning Aid System (Instrumedics, Hackensack, USA). Sections from the Tissue Microarray block were used for immunohistochemistry. The number of punches per patient ranged from 1 to 3 for both normal tissue and carcinoma and from 1 to 5 for patients with adenomas. When more than one punch was obtained, the additional punches were taken from different representative blocks. One punch was collected per patient on average (median 1.0) for each of the tissue types.

\section{Histology and Immunophenotyping}

All tissue samples were reviewed by two experienced surgical pathologists (PR and LMT) based exclusively on $\mathrm{H} \& \mathrm{E}$ staining. Adenomas were graded and carcinomas were subclassified according to the criteria published by the AFIP. ${ }^{15}$ Mild dysplasia was designated low-grade dysplasia, whereas moderate and severe dysplasia was considered high-grade dysplasia. Carcinomas essentially indistinguishable from colorectal carcinomas were classified as intestinal types, whereas carcinomas showing a dense desmoplastic stroma surrounding small glands or solid nests of tumor cells were referred to as the pancreatico-biliary subtype. Invasive papillary carcinomas typically formed papillary and micropapillary structures in their invasive component and poorly differentiated adenocarcinomas lacked histologic features of glandular or other differentiation. Additionally, an intestinalmucinous subtype, characterized by any mucinous differentiation, was defined. For immunohistochemistry, sections were pre-treated with CC1 (Ventana Medical Systems, Tucson, AZ, USA) and incubated with primary antibodies against CD24, P-cadherin and S100A4. Characteristics of antibodies are listed in Table 1. Staining procedures were performed on a 
Table 1 Characteristics of antibodies

\begin{tabular}{llll}
\hline Antibody & Dilution & Clone & Manufacturer \\
\hline S100A4 & $1: 1000$ & Polyclonal & Dako \\
P-cadherin & $1: 50$ & 56 & BD Biosciences \\
CD24 & $1: 200$ & SN3b & Neomarkers \\
\hline
\end{tabular}

Benchmark immunohistochemistry staining system (Ventana Medical Systems, Tucson, AZ, USA) using iVIEW-DAB as chromogen.

\section{Evaluation of Immunohistochemistry}

Immunoreactivity for each protein marker was scored semi-quantitatively by evaluating the number of positive cells over the total number of cells. Lack of immunoreactivity was scored as negative (Score 0 ), positivity in less than $10 \%$ of cells as weakly positive (Score 1) and in more than $10 \%$ of cells as strongly positive (Score 2). Single cells showed a cytoplasmatic CD24 positivity while the vast majority of positive cases was membranously positive. For statistical reasons only membranous CD24 immunoreactivity was evaluated. S100A4 showed a cytoplasmatic and P-cadherin both a membranous and a cytoplasmatic staining pattern which were evaluated separately (P-cadherin (mem) and P-cadherin (cyt)).

\section{Statistical Analyses}

Differences in protein expression between normal, adenoma and carcinoma were evaluated by the Chi-Square test. A Bonferroni correction for multiple comparisons was performed. Consequently, only $P$-values $<0.013$ were considered statistically significant. (SAS Version 9.1, NC, USA). Differences in expression between the tumor types were also analyzed. In order to increase the sample size of individual stratifications, staining of 0 was compared to staining of degree 1 and 2 combined. To determine the optimal combination of markers able to correctly classify patients with normal tissue, adenoma and carcinoma, classification and regression tree analysis was used (DTREG Predictive Modeling Software, www.dtreg.com). With this method, a clinical decision rule is generated and output in the form of an interpretable decision-tree. The computer-generated algorithm uses a binary recursive process which splits the data into the best possible combination of variables to optimally classify patients with or without a particular outcome. To measure the classification error as a function of tree size, 10-fold cross-validation experiments were performed. Tissues with missing values were excluded from the analysis. Classification and regression tree analysis applied to normal tissue and adenoma resulted in a final tree of 3 terminal nodes and a misclassification rate of $27 \%$. For the classification of normal tissue and carcinoma, the prunedtree had 3 terminal nodes and a misclassification rate of $21 \%$ and for adenoma and carcinoma, the final tree had 3 terminal nodes and misclassification rate of $33 \%$. Finally, the positive predictive value and negative predictive value for each marker and combination were obtained.

\section{Results}

\section{Histologic Classification of Ampullary Carcinomas}

Classification of 177 ampullary carcinomas investigated in our series identified 88 intestinal types (50\%), 41 pancreatico-biliary types (23\%), 23 poorly differentiated adenocarcinomas $(13 \%), 16$ intestinal-mucinous types $(9.0 \%)$ and 9 invasive papillary types $(5 \%)$.

\section{Grading of Ampullary Adenomas and Association to Carcinoma Subtypes}

Histologic grading was evaluable in 78/114 (68\%) adenomas in our series and included 59/78 (76\%) cases of low-grade dysplasia and 19/78 (24\%) cases with high-grade dysplasia. All adenomas demonstrated tubular or tubulovillous architecture. 84/114 (74\%) adenoma samples were derived from patients suffering from coexisting carcinoma. The corresponding subtypes were 54 intestinal types, 13 intestinal-mucinous types, 7 pancreatico-biliary types, 8 poorly differentiated adenocarcinomas and 2 invasive papillary types.

\section{Expression of S100A4, P-Cadherin and CD24 in Ampullary Carcinomas, Adenomas and Normal Mucosa}

The expression of all investigated marker proteins differed significantly between the carcinoma, adenoma and normal mucosa samples (Table 2). Only $1 / 122$ evaluable normal tissue samples (1\%) showed strong expression of S100A4 and less than $7 \%$ of cases demonstrated strong immunoreactivity against P-cadherin or CD24 (Figure 1).

Tissue samples with no missing values for any of the 3 markers were entered into classification and regression tree analysis. Of the markers analyzed, only P-cadherin (mem) and S100A4 were found to discriminate between normal mucosa and adenoma (Figure 2) as well as between normal mucosa and carcinoma samples (Figure 3) with membranous immunoreactivity of P-cadherin observed more frequently in adenomas (86\%) and carcinomas (93\%) compared with normal mucosa samples. The positive predictive value of $\mathrm{P}$-cadherin for adenoma was $42 \%$ and for carcinoma was $59 \%$ while the negative predictive value was $95 \%$ in both cases. In the case of P-cadherin (mem) negativity, 
Table 2 Immunoprofiles of normal mucosa, adenoma and carcinoma tissue samples

\begin{tabular}{|c|c|c|c|c|c|}
\hline Marker & Expression & Normal, N (\%) & Adenoma, N (\%) & Carcinoma, N (\%) & $\mathrm{P}$-value \\
\hline \multirow[t]{3}{*}{ S100A4 } & 0 & $105(86)$ & $73(75)$ & $74(48)$ & \multirow[t]{3}{*}{$<0.001$} \\
\hline & 1 & 16 (13) & $13(14)$ & $30(20)$ & \\
\hline & 2 & $1(1)$ & $11(11)$ & $49(32)$ & \\
\hline \multirow[t]{3}{*}{ P-cadherin (cyt) } & 0 & $80(63)$ & $36(37)$ & $47(32)$ & \multirow[t]{3}{*}{$<0.001$} \\
\hline & 1 & 39 (31) & $44(45)$ & 79 (53) & \\
\hline & 2 & $8(6)$ & $18(18)$ & $23(15)$ & \\
\hline \multirow[t]{3}{*}{ P-cadherin (mem) } & 0 & $118(93)$ & $56(57)$ & $63(42)$ & \multirow[t]{3}{*}{$<0.001$} \\
\hline & 1 & $4(3)$ & $22(23)$ & $41(28)$ & \\
\hline & 2 & $5(4)$ & $20(20)$ & $45(30)$ & \\
\hline \multirow[t]{3}{*}{ CD24 } & 0 & $75(60)$ & $58(62)$ & $69(45)$ & \multirow[t]{3}{*}{$<0.001$} \\
\hline & 1 & $43(34)$ & 36 (38) & $55(36)$ & \\
\hline & 2 & $7(6)$ & $0(0)$ & 28 (19) & \\
\hline
\end{tabular}

strong expression of S100A4 was predictive of neoplastic lesions (adenomas 100\%, carcinomas $100 \%$ ) although the number of cases was small (Figures 2 and 3). Comparing adenomas and carcinomas, S100A4 demonstrated a positive and negative predictive value of 55 and $79 \%$, respectively for carcinoma. Negativity of the marker and strong expression of CD24 was exclusively found in carcinomas $(100 \%$, Figure 4$)$. The positive and negative predictive value for this combination of markers was 12 and $100 \%$, respectively. In the case of S100A4 positivity, 77\% of samples were carcinomas compared to $23 \%$ adenomas (Figure 4 ).

\section{Expression of S100A4, P-Cadherin and CD24 in Ampullary Carcinoma Subtypes}

The expression of P-cadherin and CD24 did not differ significantly between the carcinoma subtypes. Immunoreactivity against S100A4, however, showed a borderline $P$-value $(0.041)$, but since the number of cases varied markedly between the distinct subgroups this result might be of limited significance (Table 3).

\section{Correlation of S100A4, P-Cadherin and CD24 Expression with Clinical-Pathological Parameters of Carcinoma Samples}

The expression of all investigated marker proteins did not show significant differences according to T-, $\mathrm{N}$ - and M-stage or tumor grading (data not shown).

\section{Discussion}

Most ampullary carcinomas are thought to originate from pre-existing adenomas, indicating an adenoma-carcinoma sequence with stepwise accumulation of genetic alterations. ${ }^{4,5,29-31}$ In our series, $74 \%$ of patients with ampullary carcinomas had evidence of coexisting adenomas which is in line with the literature reporting synchronous lesions in 30-91\% of cases. ${ }^{4,5}$ Differentiating normal mucosa with reactive atypia from dysplasia or dysplasia from invasive carcinoma can be challenging or even impossible in bioptic material. Markers for ampullary dysplasia or invasive carcinoma could therefore be of great diagnostic value.

The cadherins are a family of glycoproteins which mediate calcium dependent homophilic cell-cell adhesion. Altered or absent expression results in decreased adhesion and might be important in tumorigenesis, particulary tumor cell invasion..$^{32,33}$ Expression of P-cadherin is physiologically restricted to cytotrophoblastic placental cells (placenta-cadherin), the basal layers of stratified epithelia in the prostate and the skin and myoepithelial cells in the breast. ${ }^{34}$ However, aberrant expression has been observed in several malignant tumors, including adenocarcinomas of the cervix, ovarian cancer, oral squamous cell carcinomas and carcinomas of the breast. ${ }^{19,22,23}$ Interestingly, also intrahepatic cholangiocarcinomas were shown to express P-cadherin (13/23 cases), whereas normal mucosa samples were consistently negative. ${ }^{26}$ In our series, membranous expression of P-cadherin was found in only $9 / 127(7 \%)$ evaluable normal mucosa samples but in more than $40 \%$ of adenomas and almost $60 \%$ of carcinomas, indicating a role in the carcinogenesis of ampullary tumors.

S100A4 is a calcium binding protein belonging to the S100 proteins which are involved in cell motility, cell adhesion and proliferation. ${ }^{35}$ Furthermore, S100A4 was reported to contribute to invasive growth of tumors, tumor progression and metastases. ${ }^{35}$ Consequently, several carcinomas were shown to express S100A4, including pancreatic carcinomas, bladder cancer and breast carcinoma. ${ }^{36-38}$ Zhao and colleagues found S100A4 expression in a small series of extrahepatic cholangiocarcinomas (8/10 cases) and biliary high-grade dysplasia (5/6 cases) but not in any of the investigated normal mucosa samples (0/10 cases). ${ }^{28}$ In our 

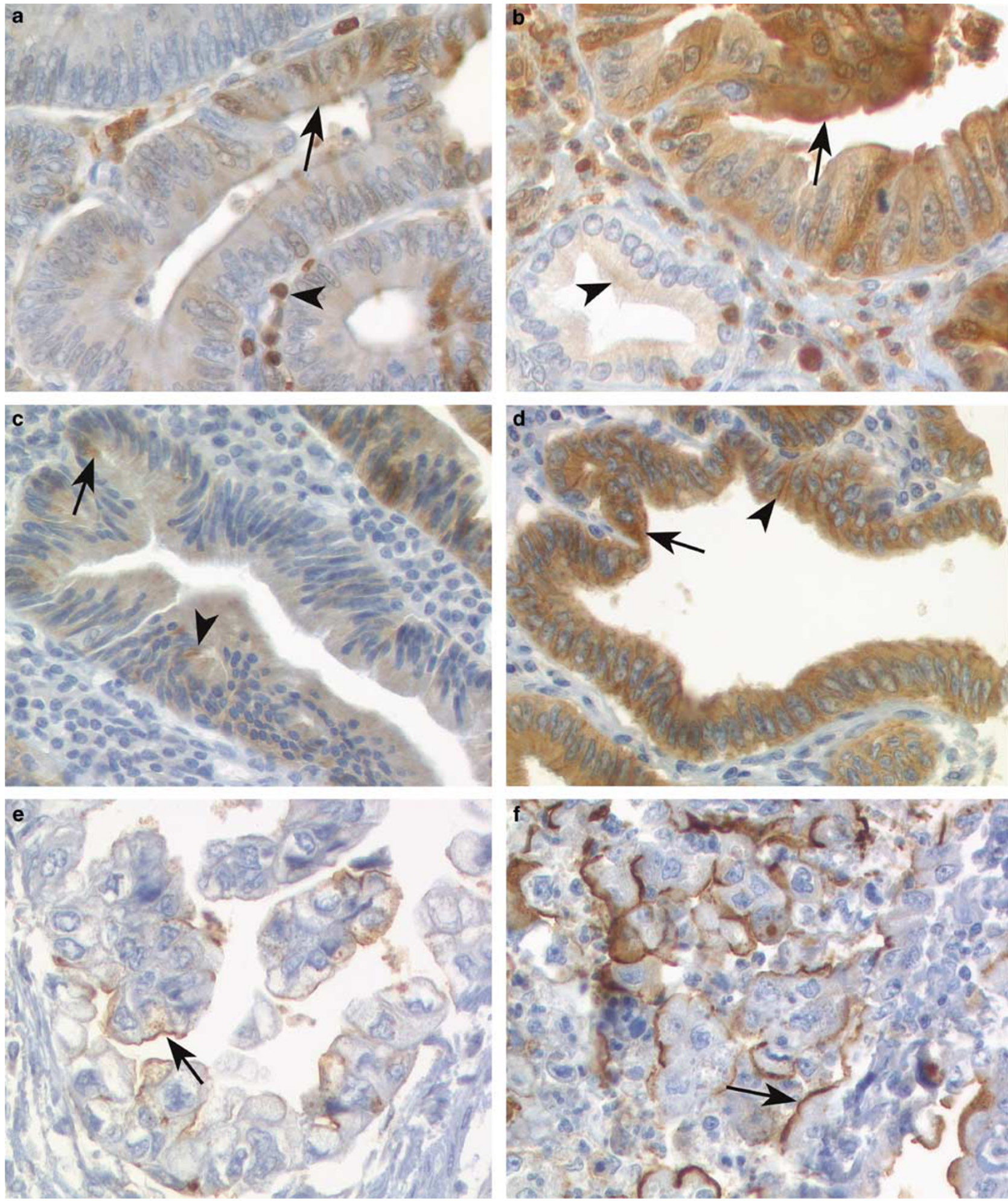

Figure 1 (a) Adenoma showing weak (Score 1) cytoplasmatic positivity for S100A4 (arrow) while mononuclear cells are strongly positive (Score 2, arrowhead). (b) Carcinoma strongly positive (Score 2) for S100A4 (arrow), normal epithelium weakly positive (Score 1) for S100A4 (arrowhead). Cytoplasmatic (arrow) and membranous (arrowhead) P-cadherin expression in Adenoma (c, weak, Score 1) and in Carcinoma (d, strong, Score 2). Carcinoma with weak (e, Score 1) and strong (f, Score 2) CD24 positivity. (All pictures $\times 200)$.

series, strong immunoreactivity against S100A4 was also virtually absent in normal mucosa samples $(1 / 122,1 \%)$. In adenomas and carcinomas, however, strong S100A4 expression was observed with increasing frequency ( $11 \%$ and $32 \%$ ) but not as commonly as in the series by Zhao et al. ${ }^{28}$ 
CD24 is a small, heavily glycosylated cell surface protein which is involved in the regulation of cell proliferation and cell-cell interaction and was

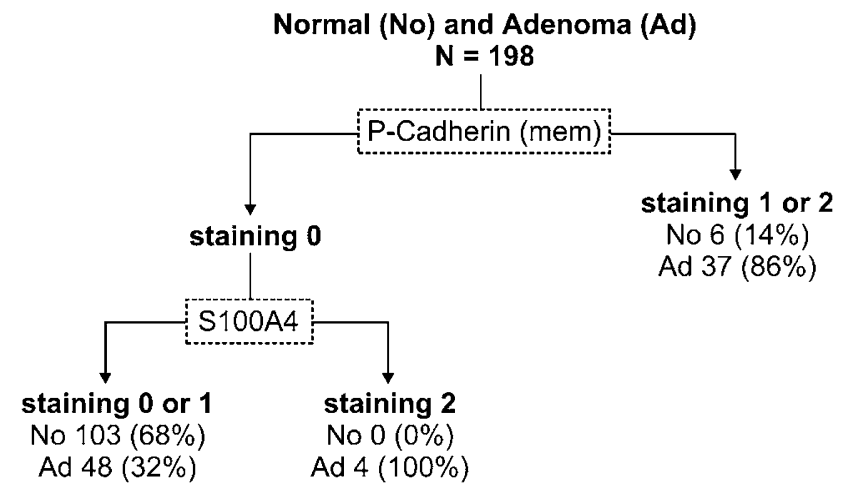

Figure 2 Decision tree to discriminate between normal mucosa and adenoma. (10-fold cross-validation; misclassification rate: $27 \%)$.

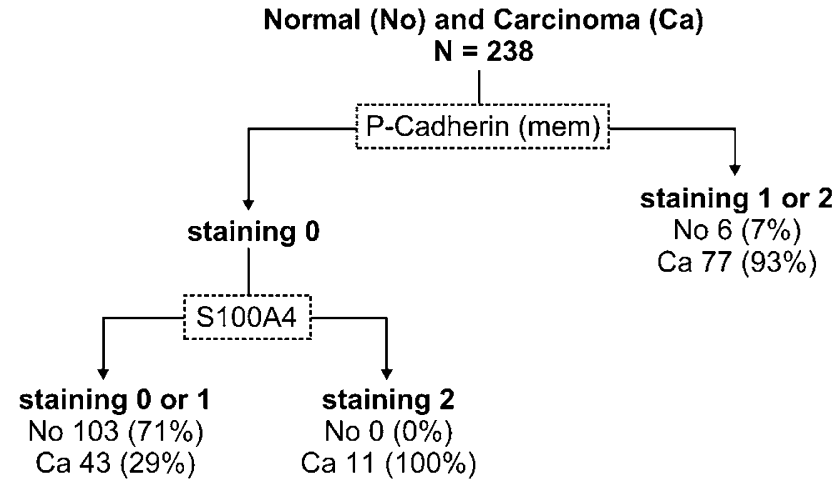

Figure 3 Decision tree to discriminate between normal mucosa and carcinoma. (10-fold cross-validation; misclassification rate $20 \%)$. shown to be expressed in several malignant tumors, including breast carcinoma, prostate cancer, non-small cell lung cancer and bladder carcinoma. ${ }^{20,21,24,39}$ Interestingly, it has only recently been suggested as a cancer stem cell marker in the gastrointestinal tract and in the liver. ${ }^{40,41}$ The only known ligand for CD24 is P-selectin which mediates binding to platelets and endothelial cells and therefore could enhance the formation of tumor thrombi and metastases. ${ }^{39}$ Also in cholangiocarcinomas and pancreatic carcinomas expression of CD24 has been reported in $51-81 \%$ of cases. ${ }^{25,27,42}$ In colorectal adenomas and carcinomas, furthermore, immunoreactivity against CD24 was observed in more than $85 \%$ of cases, but only in $17 \%$ of normal mucosa samples. ${ }^{24}$ In our series, strong expression of CD24 was mainly observed in carcinoma samples, but was rare in normal mucosa $(7 / 125,6 \%)$ and even absent in dysplastic lesions.

Since the distinct subtypes of ampullary carcinomas are known to exhibit significant differences in clinical outcome with intestinal and intestinal-

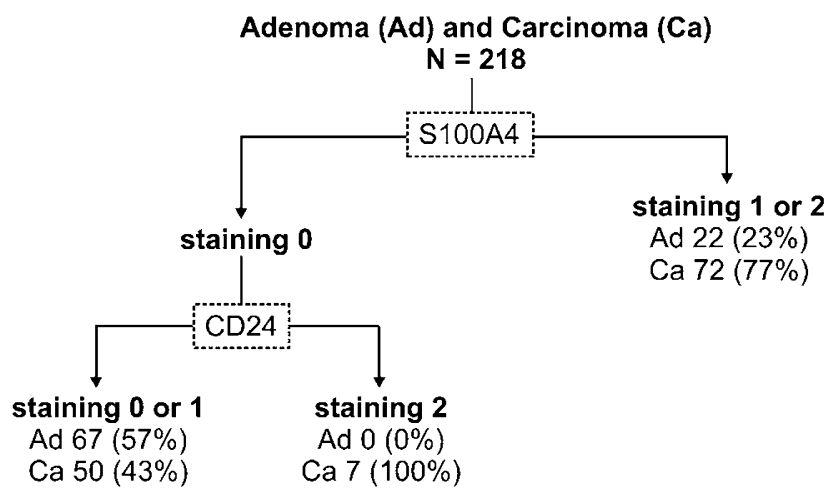

Figure 4 Decision tree to discriminate between adenoma and carcinoma. (10-fold cross-validation; misclassification rate: $33 \%$ ).

Table 3 Immunoprofiles of carcinoma subtypes

\begin{tabular}{|c|c|c|c|c|c|c|c|}
\hline Marker & Expression & $I T, \mathrm{~N}(\%)$ & $I M T, \mathrm{~N}(\%)$ & $P B T, \mathrm{~N}(\%)$ & $G 3 A C, \mathrm{~N}(\%)$ & $I P T, \mathrm{~N}(\%)$ & P-value* \\
\hline \multirow[t]{3}{*}{ S100A4 } & 0 & $44(56)$ & $5(42)$ & $9(28)$ & $8(40)$ & $6(75)$ & \multirow{3}{*}{0.041} \\
\hline & 1 & $13(16)$ & $3(25)$ & $7(22)$ & $5(25)$ & $2(25)$ & \\
\hline & 2 & $22(28)$ & $4(33)$ & $16(50)$ & $7(35)$ & $0(0)$ & \\
\hline \multirow[t]{3}{*}{ P-cadherin (cyt) } & 0 & $25(31)$ & $3(23)$ & $10(33)$ & $6(33)$ & $3(38)$ & \multirow{3}{*}{0.957} \\
\hline & 1 & $43(55)$ & $7(54)$ & $15(50)$ & $10(56)$ & 3 (38) & \\
\hline & 2 & $11(14)$ & $3(23)$ & $5(17)$ & $2(11)$ & $2(25)$ & \\
\hline \multirow[t]{3}{*}{ P-cadherin (mem) } & 0 & $37(48)$ & $5(39)$ & $9(30)$ & $8(44)$ & $2(25)$ & \multirow{3}{*}{0.437} \\
\hline & 1 & $22(28)$ & $6(46)$ & $10(33)$ & 2 (11) & 1 (13) & \\
\hline & 2 & $19(24)$ & $2(15)$ & $11(37)$ & $8(44)$ & $5(63)$ & \\
\hline \multirow[t]{3}{*}{$\mathrm{CD} 24$} & 0 & $40(51)$ & $4(33)$ & $12(60)$ & $7(35)$ & $5(71)$ & \multirow{3}{*}{0.201} \\
\hline & 1 & $32(41)$ & $8(67)$ & $8(40)$ & 7 (35) & $0(0.0)$ & \\
\hline & 2 & $6(8)$ & $0(0.0)$ & $0(0.0)$ & $6(30)$ & $2(29)$ & \\
\hline
\end{tabular}

G3AC, poorly differentiated adenocarcinoma; IMT, intestinal-mucinous type; IT, intestinal type; IPT, invasive papillary type; PBT, pancreaticobiliary type.

${ }^{*} \chi^{2}$-test to analyze staining 0 vs 1 and 2 . 
mucinous types demonstrating a favourable prognosis (cumulative survival (CS) 97 and 76 months) compared to invasive papillary types, pancreaticobiliary types and poorly differentiated adenocarcinomas (CS 59, 41 and 19 months) (Ruemmele et al, submitted), accurate classification is of crucial importance. In our series intestinal $(88 / 177,50 \%)$ and pancreatico-biliary types $(41 / 177,23 \%)$ were the most common subtypes, followed by poorly differentiated adenocarcinomas (23/177, 13\%), intestinal-mucinous types $(16 / 177,9 \%)$ and invasive papillary types $(9 / 177,5 \%)$. This is in line with most of the reported series in the literature with only several smaller studies demonstrating pancreaticobiliary types to be more common than intestinal types. ${ }^{14,15,17,18}$ The expression of the investigated marker proteins, however, did not differ significantly between the distinct subgroups and therefore do not seem to be useful in subclassification of ampullary carcinomas.

Taken together, S100A4, P-cadherin and CD24 are primarily expressed in neoplastic ampullary lesions and only rarely in normal mucosa samples. Strong expression of CD24 might, furthermore, help to differentiate adenoma from invasive carcinoma. Since a large amount of neoplastic samples are negative for all the investigated marker proteins, this panel of cell adhesion proteins can not replace conventional light microscopy but, in case of immunoreactivity, can support and confirm the diagnosis of neoplastic disease.

\section{Disclosure/conflict of interest}

We declare that we have no conflict of interest.

\section{References}

1 Albores-Saavedra J, Scoazec JC, Wittekind C, et al. Tumours of the gallbladder and extrahepatic ducts. In: Hamilton SR, Aaltonen LA (eds). World Health Organization Classification of Tumours: Pathology and Genetics of Tumours of the Digestive System, Vol. IARC Press: Lyon (France), 2000, pp 203-218.

2 Neoptolemos JP, Talbot IC, Carr-Locke DL, et al. Treatment and outcome in 52 consecutive cases of ampullary carcinoma. Br J Surg 1987;74:957-961.

3 Wright NH, Howe JR, Rossini FP, et al. Tumours of the small intestine. In: Hamilton SR, Aaltonen LA (eds). World Health Organization Classification of Tumours: Pathology and Genetics of Tumours of the Digestive System, Vol. IARC Press: Lyon (France), 2000, pp 69-92.

4 Kaiser A, Jurowich C, Schonekas H, et al. The adenoma-carcinoma sequence applies to epithelial tumours of the papilla of Vater. Z Gastroenterol 2002;40:913-920.

5 Takashima M, Ueki T, Nagai E, et al. Carcinoma of the ampulla of Vater associated with or without adenoma: a clinicopathologic analysis of 198 cases with reference to p53 and Ki-67 immunohistochemical expressions. Mod Pathol 2000;13:1300-1307.
6 Todoroki T, Koike N, Morishita Y, et al. Patterns and predictors of failure after curative resections of carcinoma of the ampulla of Vater. Ann Surg Oncol 2003;10:1176-1183.

7 Di Giorgio A, Alfieri S, Rotondi F, et al. Pancreatoduodenectomy for tumors of Vater's ampulla: report on 94 consecutive patients. World J Surg 2005;29: 513-518.

8 Howe JR, Klimstra DS, Moccia RD, et al. Factors predictive of survival in ampullary carcinoma. Ann Surg 1998;228:87-94.

9 Sperti C, Pasquali C, Piccoli A, et al. Radical resection for ampullary carcinoma: long-term results. Br J Surg 1994;81:668-671.

10 Talamini MA, Moesinger RC, Pitt HA, et al. Adenocarcinoma of the ampulla of Vater. A 28-year experience. Ann Surg 1997;225:590-599; discussion 9-600.

11 Bouvet M, Gamagami RA, Gilpin EA, et al. Factors influencing survival after resection for periampullary neoplasms. Am J Surg 2000;180:13-17.

12 Chang MC, Chang YT, Tien YW, et al. Distinct chromosomal aberrations of ampulla of Vater and pancreatic head cancers detected by laser capture microdissection and comparative genomic hybridization. Oncol Rep 2005;14:867-872.

13 Yeo CJ, Cameron JL, Sohn TA, et al. Six hundred fifty consecutive pancreaticoduodenectomies in the 1990s: pathology, complications, and outcomes. Ann Surg 1997;226:248-257; discussion 57-60.

14 Fischer HP, Zhou H. Pathogenesis of carcinoma of the papilla of Vater. J Hepatobiliary Pancreat Surg 2004;11: 301-309.

15 Albores-Saavedra J, Henson DE, Klimstra DS. Tumors of the gallbladder, extrahepatic bile ducts, and ampulla of Vater. Armed Forces Institute of Pathology: Washington DC, 2000;259-316pp.

16 Fischer HP, Zhou H. [Pathogenesis and histomorphology of ampullary carcinomas and their precursor lesions. Review and individual findings]. Pathologe 2003;24:196-203.

17 Kimura W, Futakawa N, Yamagata S, et al. Different clinicopathologic findings in two histologic types of carcinoma of papilla of Vater. Jpn J Cancer Res 1994;85: 161-166.

18 Matsubayashi $\mathrm{H}$, Watanabe $\mathrm{H}$, Yamaguchi $\mathrm{T}$, et al. Differences in mucus and K-ras mutation in relation to phenotypes of tumors of the papilla of Vater. Cancer 1999;86:596-607.

19 Han AC, Edelson MI, Peralta Soler A, et al. Cadherin expression in glandular tumors of the cervix. Cancer 2000;89:2053-2058.

20 Kristiansen G, Schluns K, Yongwei Y, et al. CD24 is an independent prognostic marker of survival in nonsmall cell lung cancer patients. Br J Cancer 2003;88: 231-236.

21 Kristiansen G, Winzer KJ, Mayordomo E, et al. CD24 expression is a new prognostic marker in breast cancer. Clin Cancer Res 2003;9:4906-4913.

22 Lo Muzio L, Campisi G, Farina A, et al. P-cadherin expression and survival rate in oral squamous cell carcinoma: an immunohistochemical study. BMC Cancer 2005;5:63.

23 Patel IS, Madan P, Getsios S, et al. Cadherin switching in ovarian cancer progression. Int J Cancer 2003;106: 172-177.

24 Sagiv E, Memeo L, Karin A, et al. CD24 is a new oncogene, early at the multistep process of colorectal 
cancer carcinogenesis. Gastroenterology 2006;131: 630-639.

25 Agrawal S, Kuvshinoff BW, Khoury T, et al. CD24 expression is an independent prognostic marker in cholangiocarcinoma. J Gastrointest Surg 2007;11: $445-451$.

26 Obama K, Ura K, Li M, et al. Genome-wide analysis of gene expression in human intrahepatic cholangiocarcinoma. Hepatology 2005;41:1339-1348.

$27 \mathrm{Su}$ MC, Hsu C, Kao HL, et al. CD24 expression is a prognostic factor in intrahepatic cholangiocarcinoma. Cancer Lett 2006;235:34-39.

28 Zhao H, Davydova L, Mandich D, et al. S100A4 protein and mesothelin expression in dysplasia and carcinoma of the extrahepatic bile duct. Am J Clin Pathol 2007;127:374-379.

29 Kozuka S, Tsubone M, Yamaguchi A, et al. Adenomatous residue in cancerous papilla of Vater. Gut 1981;22: 1031-1034.

30 Perzin KH, Bridge MF. Adenomatous and carcinomatous changes in hamartomatous polyps of the small intestine (Peutz-Jeghers syndrome): report of a case and review of the literature. Cancer 1982;49:971-983.

31 Sellner F. Investigations on the significance of the adenoma-carcinoma sequence in the small bowel. Cancer 1990;66:702-715.

32 Takeichi M. Cadherin cell adhesion receptors as a morphogenetic regulator. Science 1991;251: 1451-1455.

33 Vleminckx K, Vakaet Jr L, Mareel M, et al. Genetic manipulation of E-cadherin expression by epithelial tumor cells reveals an invasion suppressor role. Cell 1991;66:107-119.

34 Shimoyama Y, Hirohashi S, Hirano S, et al. Cadherin cell-adhesion molecules in human epithelial tissues and carcinomas. Cancer Res 1989;49:2128-2133.

35 Mazzucchelli L. Protein S100A4: too long overlooked by pathologists? Am J Pathol 2002;160:7-13.

36 de Silva Rudland S, Martin L, Roshanlall C, et al. Association of S100A4 and osteopontin with specific prognostic factors and survival of patients with minimally invasive breast cancer. Clin Cancer Res 2006;12:1192-1200.

37 Matsumoto K, Irie A, Satoh $\mathrm{T}$, et al. Expression of S100A2 and S100A4 predicts for disease progression and patient survival in bladder cancer. Urology 2007; 70:602-607.

38 Oida Y, Yamazaki H, Tobita K, et al. Increased S100A4 expression combined with decreased E-cadherin expression predicts a poor outcome of patients with pancreatic cancer. Oncol Rep 2006;16:457-463.

39 Kristiansen G, Sammar M, Altevogt P. Tumour biological aspects of CD24, a mucin-like adhesion molecule. J Mol Histol 2004;35:255-262.

40 Lee CJ, Dosch J, Simeone DM. Pancreatic cancer stem cells. J Clin Oncol 2008;26:2806-2812.

41 Zou GM. Cancer initiating cells or cancer stem cells in the gastrointestinal tract and liver. J Cell Physiol 2008; e-pub ahead of print.

42 Jacob J, Bellach J, Grutzmann R, et al. Expression of CD24 in adenocarcinomas of the pancreas correlates with higher tumor grades. Pancreatology 2004;4:454-460. 